\title{
Conceptual design of the ITER fast-ion loss detector
}

\author{
M. Garcia-Munoz, ${ }^{1,2, a)}$ M. Kocan, ${ }^{3}$ J. Ayllon-Guerola,,${ }^{1,2}$ L. Bertalot, ${ }^{3}$ Y. Bonnet, ${ }^{3}$ N. Casal, ${ }^{3}$ \\ J. Galdon,, ${ }^{1,2}$ J. Garcia Lopez, ${ }^{1,2}$ T. Giacomin, ${ }^{3}$ J. Gonzalez-Martin, ${ }^{1,2}$ J. P. Gunn, ${ }^{4}$ \\ M. C. Jimenez-Ramos, ${ }^{1,2}$ V. Kiptily, ${ }^{5}$ S. D. Pinches, ${ }^{3}$ M. Rodriguez-Ramos, ${ }^{1,2}$ R. Reichle, ${ }^{3}$ \\ J. F. Rivero-Rodriguez, ${ }^{1,2}$ L. Sanchis-Sanchez, ${ }^{1,2}$ A. Snicker, ${ }^{6}$ G. Vayakis, ${ }^{3}$ E. Veshchev, ${ }^{3}$ \\ Ch. Vorpahl, ${ }^{3}$ M. Walsh, ${ }^{3}$ and R. Walton ${ }^{3}$ \\ ${ }^{1}$ Department of Atomic, Molecular and Nuclear Physics, University of Seville, 41012 Seville, Spain \\ ${ }^{2}$ CNA (Universidad de Sevilla-CSIC-J. Andalucía), Seville, Spain \\ ${ }^{3}$ ITER Organization, Route de Vinon-sur-Verdon, CS 90 046, 13067 Saint Paul-lez-Durance Cedex, France \\ ${ }^{4}$ CEA, IRFM, F-13108 Saint Paul Lez Durance, France \\ ${ }^{5} \mathrm{CCFE}$, Abingdon OX14 3DB, United Kingdom \\ ${ }^{6}$ Max-Planck-Institut für Plasmaphysik, Garching, Germany
}

(Presented 7 June 2016; received 25 June 2016; accepted 6 August 2016;

published online 30 August 2016)

\begin{abstract}
A conceptual design of a reciprocating fast-ion loss detector for ITER has been developed and is presented here. Fast-ion orbit simulations in a 3D magnetic equilibrium and up-to-date first wall have been carried out to revise the measurement requirements for the lost alpha monitor in ITER. In agreement with recent observations, the simulations presented here suggest that a pitch-angle resolution of $\sim 5^{\circ}$ might be necessary to identify the loss mechanisms. Synthetic measurements including realistic lost alpha-particle as well as neutron and gamma fluxes predict scintillator signal-to-noise levels measurable with standard light acquisition systems with the detector aperture at $\sim 11 \mathrm{~cm}$ outside of the diagnostic first wall. At measurement position, heat load on detector head is comparable to that in present devices. Published by AIP Publishing. [http://dx.doi.org/10.1063/1.4961295]
\end{abstract}

\section{INTRODUCTION}

ITER is designed to produce a self-maintained burning plasma dominated by alpha-particle heating with a fusion power amplification factor, $\mathrm{Q} \geq 10$. A good alpha-particle confinement is therefore of paramount importance for the ITER project. Fusion born alpha particles as well as other fastions generated by auxiliary heating systems are, however, subject to transport by a broad spectra of magnetohydrodynamic (MHD) perturbations. A large fast-ion redistribution/loss could have a significant impact on their plasma heating and current drive efficiency compromising the machine fusion performance. An intense, and localized, fast-ion loss could even threaten the device integrity damaging some plasma facing components. ${ }^{1}$

Although several diagnostics for confined fast-ions are being proposed for ITER, a lost alpha diagnostic has not been approved as of yet. The harsh environment in ITERa nuclear installation-places a number of constraints on standard fast-ion loss detection techniques unprecedented in present tokamaks with easier access and more tolerable conditions.

On the basis of the physics requirements, the ITPA Energetic Particle (EP) Topical Group (TG) has started to undertake a conceptual study of four different and complementary fastion loss detectors for ITER: a reciprocating Fast-Ion Loss

Note: Contributed paper, published as part of the Proceedings of the 21st Topical Conference on High-Temperature Plasma Diagnostics, Madison, Wisconsin, USA, June 2016.

a) Author to whom correspondence should be addressed. Electronic mail: mgm@us.es
Detector (FILD),, 3 a fast-ion loss monitor based on edge gamma radiation, an under-the-dome detector, and a dedicated infrared system. Based on the ITPA EP TG prioritization, the Port Plugs and Diagnostics Integration Division at ITER Organization has recently initiated an effort to develop a conceptual design of a reciprocating FILD in ITER. We present here the physics basis for this selection of diagnostics for the ITER loss alpha diagnostic as well as the conceptual design of a reciprocating FILD system including the expected velocity-space coverage. $\mathrm{ASCOT}^{4}$ simulations are used to estimate the fast-ion flux to the FILD head inserted into the ITER far Scrape-Off Layer (SOL), taking into account the shaping of the surrounding first wall panels as well as 3D equilibrium including externally applied Resonant Magnetic Perturbations (RMPs).

In light of the project needs and actual capabilities of the proposed techniques, the specifications for the lost alpha monitors in ITER will be discussed in Section II. Section III is devoted to the conceptual design of a reciprocating FILD system for ITER including heat load analysis, neutronic, and expected synthetic signals.

\section{MEASUREMENT REQUIREMENTS}

The measurement requirements for fast-ion losses in ITER have been revised on several occasions based on the current fast-ion understanding and expected diagnostic capabilities. ${ }^{5}$ Given the state-of-the-art, we propose here to update the current measurement requirements introducing velocity-space resolution as well as increasing the temporal resolution. 
Recent time-resolved phase-space measurements of MHD induced fast-ion losses have provided important insight into the wave-particle interaction responsible for the underlying transport mechanisms. Accurate velocityspace measurements of fast-ion losses induced by Alfven Eigenmodes (AEs) and Energetic Particle Modes (EPMs) have allowed identifying the wave-particle resonances responsible for the observed convective and diffusive fast-ion losses. ${ }^{6,7}$ Similarly, multiple accurate velocity-space measurements of fast-ion losses induced by externally applied RMPs have revealed the impact the spatial structure of the applied 3D fields has on the escaping ion phase-space. ${ }^{8,9}$

Simulations of alpha particle losses in the ITER 15 MA scenario with a 3D equilibrium, including Toroidal Field (TF) ripple, ferritic inserts (FIs) and $n=4$ RMP (perturbation fields calculated in vacuum), and an up-to-date 3D wall have been carried out with the ASCOT code to guide the revision of the measurement requirements presented here. All ASCOT simulations presented here have been carried out using the guiding center approach for particles well confined. The orbit integrator in ASCOT switches from guiding center to full orbit when the particle is near the wall. Fig. 1 shows the expected alpha particle heat load on the ITER first wall. The total number of $10^{6}$ test fusion born alpha-particles have been followed until they thermalize or hit the wall. The different wall structures of the low field side are clearly visible together with the $n=4$ RMP structure. As expected, due to the ion gradB drift, most losses appear at the divertor and mid-plane wall structures with a maximum heat load around $1 \mathrm{MW} / \mathrm{m}^{2}$. This heat load comprises approximately $12.5 \%$ of alpha particle losses and $\sim 8 \%$ of alpha particle power loss. The $n=4$ RMP, caused by the ELM mitigation coils with $\mathrm{I}_{\text {coil }}=90 \mathrm{kA}$, has been used here to simulate an extreme case with high but realistic alpha particle losses. The coupling of an external, not sufficiently shielded, RMP to an internal perturbation could eventually lead to even higher fluxes. This gives, however, an estimate of the expected alpha particle fluxes and phase-space distribution, a crucial information to define the measurement requirements and estimate the performance of the proposed diagnostics.

Based on the state-of-the-art in fast-ion physics and diagnostics, the temporal and phase-space measurement

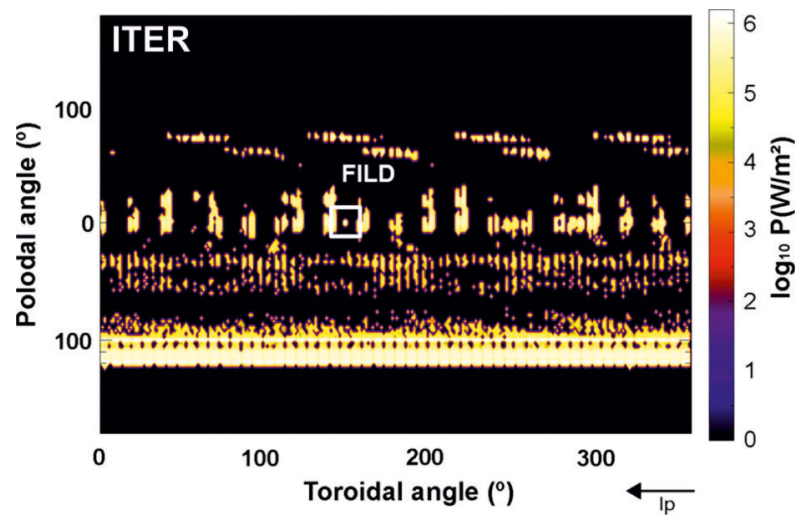

FIG. 1. ASCOT simulations of alpha particle heat load in ITER on a 3D first wall due to an externally applied $n=4$ RMP. The location of the FILD head is indicated with a white box. requirements for a lost alpha detector (or combination of detectors) are briefly discussed here.

\section{A. Temporal resolution}

A fast-ion loss detector with Alfvénic temporal resolution is highly recommended in order to identify the MHD fluctuations responsible for the measured fast-ion losses in a sea of MHD fluctuations. A MHz temporal resolution is set by the Alfvénic time, $\tau_{\mathrm{A}}=\mathrm{R}_{0} / \mathrm{v}_{\mathrm{A}} \sim 1 \mu \mathrm{s}$. This is obtained for a standard ITER DT case with an Alfvén speed $\mathrm{v}_{\mathrm{A}}=8 \times 10^{6}$ $\mathrm{m} / \mathrm{s}$ calculated with $\mathrm{B}_{\mathrm{T}}=5.2 \mathrm{~T}$, a 50\%-50\% D-T mixture and an electron density, $\mathrm{n}_{\mathrm{e}}=8 \times 10^{19} \mathrm{~m}^{-3}$.

\section{B. Spatial resolution}

The heat load spatial pattern shown in Fig. 1 gives an estimate of the spatial resolution the fast-ion loss detector should have in ITER. A spatial resolution similar to the gyroradius of the fusion born alpha-particles, $\mathrm{r}_{\mathrm{L}} \sim 7 \mathrm{~cm}$, seems to be required to be able to resolve the expected heat load wall structures.

\section{Velocity-space range and resolution}

Wave-particle resonances can occur over almost the entire fast-ion phase-space. In order to identify the wave-particle resonances responsible for the actual fast-ion transport/loss, a wide velocity-space coverage and reasonably good energy and pitch-angle resolution are thus necessary. Fig. 2 shows the velocity-space of the escaping ions collected at the head of the FILD system with its aperture located $11 \mathrm{~cm}$ outside of the Diagnostic First Wall (DFW) in the ASCOT simulations presented here. The losses appear over the entire energy range, from $\sim 6.5 \mathrm{~cm}$ (corresponding to the alpha-particle birth energy) down to the thermal energy at large pitch-angles, $\sim 80^{\circ}$. Similarly, a broad escaping ion pitch-angle range, from $40^{\circ}$ to $85^{\circ}$, with an energy similar to the birth energy, appears in the simulated losses at the FILD system. As in recent experimental observations, clear structures at certain pitchangles are observed.

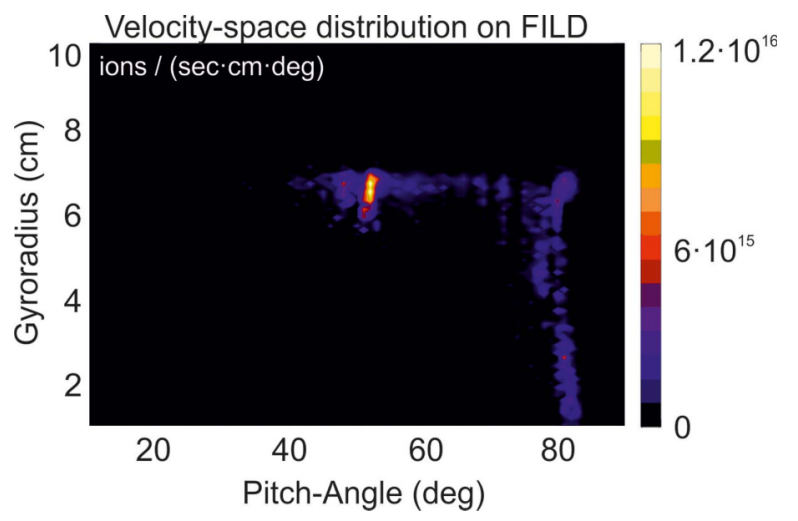

FIG. 2. ASCOT simulations. Velocity-space of alpha particle losses hitting the FILD detector head in the presence of an externally applied $n$ $=4$ RMP. 


\section{Energy coverage and resolution}

A fast-ion loss detector in ITER should cover the entire energy range from a few times the thermal energy, $\sim 0.1 \mathrm{MeV}$, up to the highest energies expected in the distribution function of fusion products and Ion Cyclotron Resonant Heating (ICRH), $\sim 10 \mathrm{MeV}$. A $10 \%$ energy resolution seems to be required to be able to monitor the dynamics of the fast-ion distribution in the presence of MHD perturbations.

\section{E. Pitch-angle coverage and resolution}

Recent observations in present tokamaks as well as the simulations presented here (Fig. 2) suggest that a pitch-angle resolution $<5 \%$ might be necessary to resolve the pitch-angle structures arising from single wave-particle resonances. Fig. 2 shows that this is especially the case for large pitch-angles corresponding to trapped orbits.

\section{THE ITER FILD SYSTEM}

A conceptual design of a reciprocating FILD for ITER has been carried out and is presented here. As a charge particle collector FILD systems must work a few gyroradii away from the separatrix and are, therefore, exposed to a relatively high thermal heat load that limits the detector operational window. The optimal detector position and measurement cycle are thus given by the balance between the measured fast-ion signals and the heat load on the detector head. Based on the ASCOT simulations presented in Sec. II, a stroke of $20 \mathrm{~cm}$ is sufficient to keep the entire detector head behind the DFW in a safe parking position as well as to deploy the detector aperture $11 \mathrm{~cm}$ outside of the DFW during measurements. In the present design, the scintillator plate is located approximately $2 \mathrm{~cm}$ behind the heat shield.

\section{A. Velocity-space coverage}

Backwards ASCOT simulations have been carried out to identify the optimal radial position of the detector head in the ITER Equatorial Port 8 (EP8). Fig. 3(a) shows the fraction of particles that, starting on the FILD aperture and followed backwards in time, make it all the way back to the plasma, i.e., escaping EP8, as a function of the aperture radial position. For $\Delta_{\text {FILD-port }}>11 \mathrm{~cm}$, the full pitch-angle range would be covered with a reasonable fraction of detected particles. Here $\Delta_{\text {FILD-port }}$ is defined as the distance between the aperture (starting point of particles in simulations) and the Diagnostic First Wall (DFW). At this position, the detector front side is $11 \mathrm{~cm}$ away from the separatrix in the standard 15 MA scenario and $13 \mathrm{~cm}$ in the ICRH optimized scenario (plasma shifted outwards by $4 \mathrm{~cm}$ to improve ICRH coupling), see Fig. 3(b). Figs. 3(c)-3(e) show some typical trajectories of escaping alphas with $3.5 \mathrm{MeV}$ followed backwards in time from the detector head with different pitch-angles for 3 different detector head radial positions; $\Delta_{\text {FILD-port }}=11,13$, and $14 \mathrm{~cm}$.
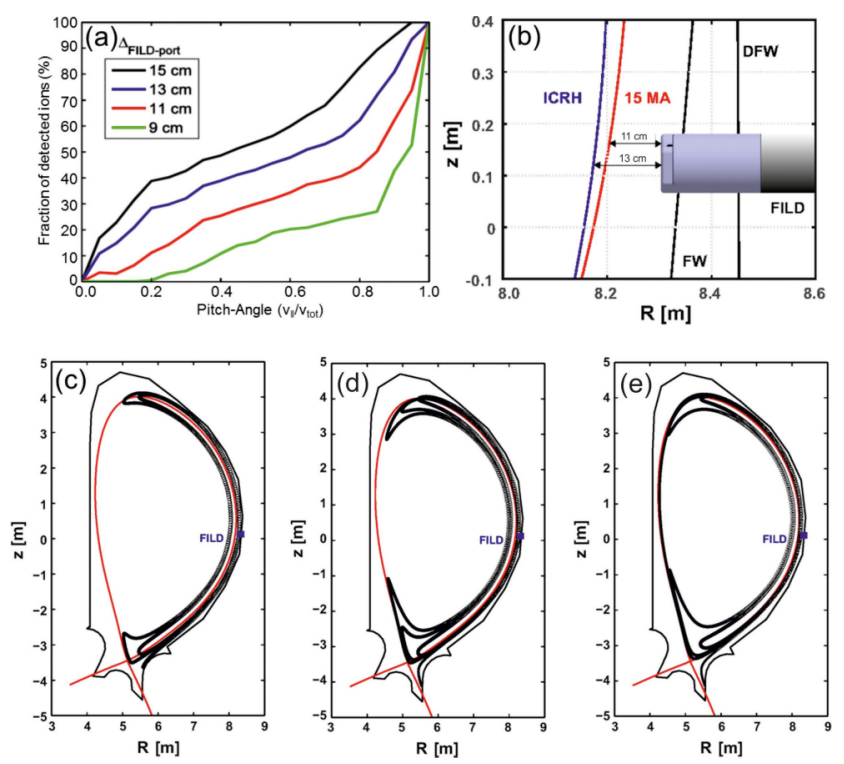

FIG. 3. ASCOT simulations. Backwards full orbit simulations. (a) Pitchangle coverage at different detector head radial positions. (b) Optimal detector head position with respect to separatrix for the standard and ICRH optimized 15 MA scenario. (c)-(e) Test orbits of $3.5 \mathrm{MeV}$ alpha particles followed backwards in time for 3 different positions of detector head; $\Delta_{\text {FILD-port }}=11,13$, and $14 \mathrm{~cm}$, respectively.

\section{B. Heat load estimate}

The heat load on the detector head due to thermal plasma has been estimated for the 15 MA scenario using the field line tracing code PFCFLUX ${ }^{10}$ and taking into account the time-averaged ELM heat flux, static inter-ELM heat flux, and plasma radiation (charge-exchange neutrals and photons). ${ }^{11}$ Fig. 4 shows the estimated heat flux on the detector head for the reference case (a) and for the same equilibrium but with the separatrix $5.5 \mathrm{~cm}$ closer to the wall/detector to simulate a worst case scenario, e.g., error in magnetic reconstruction or extreme distortion of plasma boundary by RMPs. In addition to the plasma heating calculated with the PFCFLUX code, MCNP simulations predict $\sim 10 \mathrm{~W} / \mathrm{cc}$ of volumetric nuclear heating on the detector head for the same scenario. With this total heat load and without active cooling, ANSYS predicts a detector head and scintillator plate temperature rise of only (a)

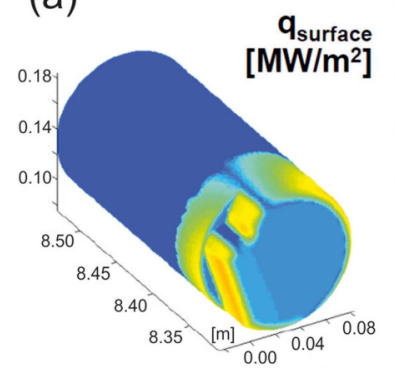

(b)

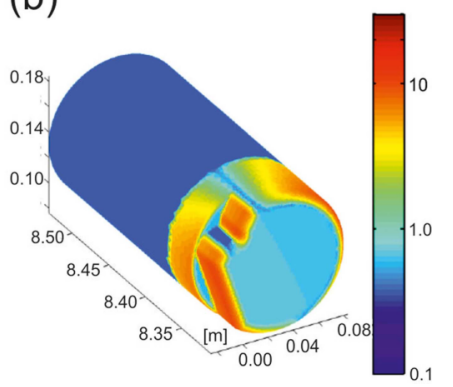

FIG. 4. Expected heat load on the FILD detector head in the ITER 15 MA scenario calculated with the field line tracing code PFCFLUX for two different separatrix positions at $\Delta_{\text {FILD-port }}=11 \mathrm{~cm}$; (a) nominal separatrix and (b) $5.5 \mathrm{~cm}$ outwards shift of the separatrix to account for the worst-case magnetic perturbation of the plasma boundary and magnetic reconstruction error. 


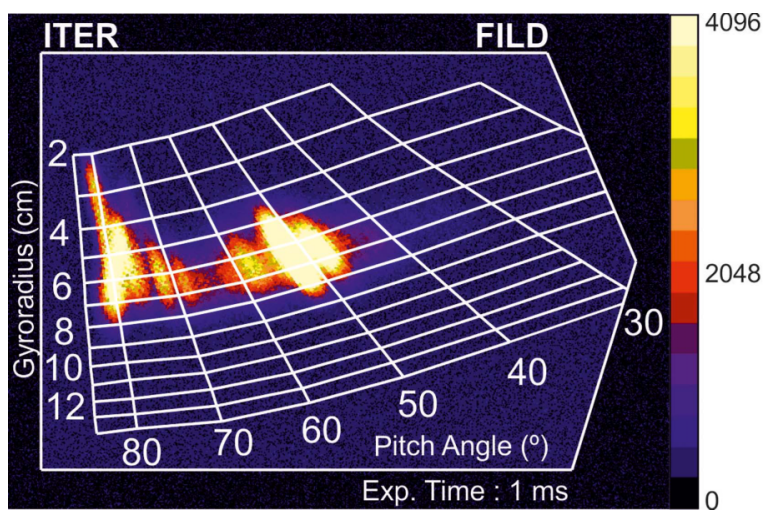

FIG. 5. Synthetic measurement of alpha particle losses induced by RMPs with the present design of the ITER FILD.

$\sim 250^{\circ} \mathrm{C}$ and $\sim 30^{\circ} \mathrm{C}$, respectively, during a measurement time of $0.2 \mathrm{~s}$.

\section{Synthetic measurement}

The FILDSIM code $^{12}$ has been used to construct a synthetic measurement taking into account the 3D detector head geometry, predicted alpha-particle flux, scintillator efficiency, and scintillator background emission caused by the MCNP simulated neutron and gamma flux in the scintillator plate. Fig. 5 shows the simulated scintillator pattern measured by a pixelfly PCO Charge Couple Device (CCD) camera. ${ }^{13}$ The signal-to-noise level and velocity-space coverage and resolution allow to measure the predicted RMP induced fastion losses on FILD, see Fig. 2, with excellent quality enabling $\mathrm{MHz}$ temporal resolution through photomultiplier arrays. The present design of the system will allow time-resolved velocityspace measurements of MHD induced alpha-particle losses in ITER.

\section{ACKNOWLEDGMENTS}

This research was supported in part by the Spanish Ministry of Economy and Competitiveness (Grant Nos. RYC2011-09152, FIS2015-69362-P, and ENE2012- 31087) and the Marie Curie FP7 Integration Grant (No. PCIG11-GA2012-321455). The views and opinions expressed herein do not necessarily reflect those of the European Commission or ITER.

${ }^{1}$ H. H. Duong et al., Nucl. Fusion 33, 749 (1993).

${ }^{2}$ S. J. Zweben et al., Nucl. Fusion 35, 1445 (1995).

${ }^{3}$ M. Garcia-Munoz et al., Rev. Sci. Instrum. 80, 053503 (2009).

${ }^{4}$ A. Snicker et al., Nucl. Fusion 53, 093028 (2013).

${ }^{5}$ F. P. Orsitto, J. M. Noterdaeme, A. E. Costley, A. J. H. Donne, and ITPA TG on Diagnostics, Nucl. Fusion 47, 1311 (2007).

${ }^{6}$ M. Garcia-Munoz et al., Phys. Rev. Lett. 104, 185002 (2010).

${ }^{7}$ G. Kramer et al., Phys. Rev. Lett. 109, 035003 (2012).

${ }^{8}$ M. Garcia-Munoz et al., Plasma Phys. Controlled Fusion 55, 124014 (2013).

${ }^{9}$ M. A. Van Zeeland et al., Plasma Phys. Controlled Fusion 56, 015009 (2014).

${ }^{10}$ M. Firdaouss et al., J. Nucl. Mater. 438, S536 (2013).

${ }^{11}$ M. Kocan et al., J. Nucl. Mater. 463, 709 (2015).

${ }^{12}$ J. Galdon et al., "The synthetic fast-ion loss detector" (unpublished).

${ }^{13}$ See http://www.pco.de/sensitive-cameras/pixelfly-qe/ for technical details of the CCD camera used in the synthetic diagnostic. 